\title{
Relationship between Poor Sleep Quality and High White Blood Cell Count in Korean Adults
}

\author{
Jae-Min Park ${ }^{1,2}$ and Ji-Won Lee \\ 'Department of Family Medicine, Gangnam Severance Hospital, Yonsei University College of Medicine, Seoul, Korea \\ 2Department of Medicine, Graduate School of Medicine, Yonsei University, Seoul, Korea
}

Objective: This study aimed to investigate the relationship between sleep quality and white blood cell (WBC) count among Korean adults. Methods: This cross-sectional study included 136 participants who attended the medical health check programs of two tertiary medical institutions from December 2019 to March 2021. Sleep quality was measured using the Korean version of the Pittsburgh Sleep Quality Index (PSQI-K). The participants were divided into the poor sleep quality group $(n=94)$ and the good sleep quality group ( $n=42)$ according to the global PSQI-K score. A high WBC count was defined as that greater than the 75th percentile in the current sample. The odds ratios (ORs) and 95\% confidence intervals (Cls) for high WBC count were calculated using multivariable logistic regression analyses after adjusting for confounding variables. Results: The mean WBC count was significantly lower in individuals with good sleep quality than in those with poor sleep quality. The prevalence of high WBC count was significantly higher in participants with poor sleep quality than in those with good sleep quality ( $p=0.018)$. Compared with the ORs (95\% Cls) in individuals with good sleep quality, the ORs (95\% Cls) for high WBC count in individuals with poor sleep quality were 3.83 (1.09-13.41) after adjusting for age, sex, alcohol consumption, coffee consumption, physical activity, marital status, hypertension, diabetes mellitus, depressive symptoms, body mass index, and homeostatic model assessment of insulin resistance. Conclusion: Poor sleep quality was positively associated with an increased risk of high WBC count among Korean adults.

Key Words: Inflammation; Leukocyte count; Sleep

Received: May 12, 2021 Revised: June 5, 2021 Accepted: June 7, 2021

Corresponding author: Ji-Won Lee, MD, PhD, Department of Family Medicine, Gangnam Severance Hospital, Yonsei University College of Medicine, 211 Eonju-ro, Gangnam-gu, Seoul 06273, Korea.

Tel: 82-2-2019-3480, Fax: 82-2-3462-8209, E-mail: indi5645@yuhs.ac

(a) This is an Open Access article distributed under the terms of the Creative Commons Attribution Non-Commercial License (https://creativecommons.org/licenses/by$\mathrm{nc/4.0)}$ which permits unrestricted non-commercial use, distribution, and reproduction in any medium, provided the original work is properly cited.

\section{INTRODUCTION}

Chronic low-grade inflammation has emerged as a central biological condition underlying the development of numerous medical disorders, including diabetes mellitus, cardiovascular diseases, neurodegenerative diseases, psychiatric disorders, and certain cancers [1,2]. Subclinical inflammation has also been associated with increased mortality [3]. The link between chronic low-grade inflammation and mortality-causing diseases indicates that the prevention of chronic low-grade inflammation is important from a public health perspective.

Sleep is a crucial factor that contributes to physical and mental health $[4,5]$. In addition, several studies have suggested that sleep quality can affect subclinical inflammation [6,7]. Although the exact mechanism between sleep and chronic low-grade inflammation is not fully understood, previous studies have shown that greater sleep disturbance was associated with higher levels of some inflammatory markers, such as interleukin-6 (IL-6) and C-reactive protein (CRP) [8-10]. In addition, several studies have revealed that experimental sleep deprivation caused an increase in the natural production of tumor necrosis factor- $\alpha$ (TNF- $\alpha$ ) and IL-6 in monocytes [11-13].

However, IL-6, TNF- $\alpha$, and CRP, which have been investigated in previous studies, are not routinely measured in clinical practice. In contrast, white blood cell (WBC) count, which is also known as a clinical marker of inflammation, is routinely tested in clinical practice. Few studies have examined the association between sleep quality and WBC count, and few studies have addressed the association between sleep quality and inflammatory markers in Koreans. Therefore, in this study, we aimed to investigate the relationship between sleep quality and WBC count in Korean adults. 


\section{METHODS}

\section{Study overview and study participants}

This was a retrospective, cross-sectional study that included volunteers who were attending the medical health check programs at two institutions, the Severance Health Checkup and the Department of Family Medicine of the Gangnam Severance Hospital, Seoul, Korea, between December 2019 and March 2021. A total of 161 adults over 19 years of age were surveyed. We excluded individuals who had missing data or did not complete the questionnaire. Since smoking could result in a raised WBC count, we also excluded current smokers. Of the remaining participants, those with a WBC count $<4,000$ cells $/ \mu \mathrm{L}$ or $>10,000$ cells $/ \mu \mathrm{L}$ were excluded to rule out the possibility of bone marrow suppressive illnesses, infections, or inflammatory disorder. After these exclusions, 136 participants ( 42 men and 94 women) were included in the final analysis. Written informed consent was obtained from each participant. This study was conducted in accordance with the ethical principles of the Declaration of Helsinki and was approved by the Institutional Review Board of Yonsei University Gangnam Severance Hospital (Institutional Review Board number: 3-2019-0226).

\section{Covariates}

Each participant completed a questionnaire that included questions regarding past medical history, marital status, smoking status, alcohol consumption, and coffee consumption. Alcohol drinker was defined as alcohol consumption on one or more days per week, and that of coffee was defined as consuming one or more cups a day. Physical activity was assessed using the Korean version of the International Physical Activity Questionnaire short form [14], and a regular exerciser was defined as a person who performed $\geq 20$ minutes of vigorous physical activity $\geq 3$ days a week or $\geq 30$ minutes of moderate physical activity $\geq 5$ days a week. Depressive symptoms were evaluated using the Beck Depression Inventory (BDI) questionnaire [15,16], and a person having depressive symptoms was defined as a person with a BDI score $\geq 10[17,18]$.

Anthropometric measurements were obtained by two trained medical staff members in accordance with the standardized procedures in each of the two hospitals. Body weight and height were measured with participants wearing light indoor clothing and no shoes. Body mass index (BMI) was calculated as body weight (kg) divided by height squared $\left(\mathrm{m}^{2}\right)$. Systolic and diastolic blood pressures were obtained from patients in the sitting position after a 10-min resting period. Blood samples were collected from patients following at least 8 hours of fasting. WBC count were measured using the XN9000 automated hematology analyzer (Sysmex, Kobe, Japan) and the ADVIA 2120i hematology analyzer (Siemens, Munich, Germany). Fasting plasma glucose was measured using the AU5800 autoanalyzer (Beckman Coulter, Brea, CA, USA). Serum insulin levels were measured using electrochemiluminescence immunoassay with Cobas E601 analyzers (Hoff-
man-La Roche, Basel, Switzerland).

The homeostasis model assessment of insulin resistance (HOMAIR) was calculated using the following formula: fasting plasma glucose $(\mathrm{mg} / \mathrm{dL}) \times$ fasting insulin $(\mu \mathrm{IU} / \mathrm{mL}) / 405$ [19].

\section{Measurement of sleep quality}

Sleep quality was measured using the Korean version of the Pittsburgh Sleep Quality Index (PSQI-K). This index is a self-administered questionnaire that includes 19 questions for assessing sleep quality over the previous month [20,21]. It is composed of seven subcategories: subject sleep quality, sleep latency, sleep duration, habitual sleep efficiency, sleep disturbance, use of sleeping medication, and daytime dysfunction. Each item in the PSQI-K is scored from 0 to 3 , where 0 means the positive extreme and 3 means the negative extreme on the Likert scale; the global PSQI-K score is the sum of these items, with scores ranging from 0 to 21 . Good sleep quality was defined as a global PSQI-K score of $\leq 5$, and poor sleep quality was defined as a global PSQI-K score $>5$.

\section{Statistical analysis}

Normal distribution was evaluated by determination of skewness using the Kolmogorov-Smirnov test. Serum insulin and HOMAIR values showed a skewed distribution. The characteristics of the study participants according to sleep quality were expressed as mean \pm standard deviation, median (interquartile range), or percentages and compared using independent t-test or Wilcoxon rank-sum test for the continuous variables and chi-squared test for the categorical variables. To evaluate the association between sleep quality and WBC count, we used multiple linear regression analyses. The odds ratios (ORs) and 95\% confidence intervals (CIs) for high WBC count (>75th percentile in the current sample) were calculated using multivariable logistic regression analysis after adjusting for the confounding variables. All analyses were conducted using the SPSS statistical software (version 25.0; IBM Corp., Armonk, NY, USA). All statistical tests were two-sided, and statistical significance was determined at a $p$-value $<0.05$.

\section{RESULTS}

Table 1 shows the characteristics of the study participants according to their sleep quality. The number of participants with good sleep quality and poor sleep quality was 94 and 42, respectively. The mean or median values of WBC count, insulin, and HOMA-IR were significantly lower in individuals with good sleep quality than in those with poor sleep quality.

Figure 1 presents the prevalence of high WBC counts according to sleep quality. The prevalence of high WBC count was significantly higher in participants with poor sleep quality than in those with good sleep quality $(\mathrm{p}=0.018)$.

Table 2 presents the results of linear regression analyses that were used to investigate the association between sleep quality and WBC count. Sleep quality showed statistically significant associations with WBC count in multiple linear regression analyses. The asso- 
Table 1. Characteristics of the study participants according to sleep quality

\begin{tabular}{|c|c|c|c|}
\hline & $\begin{array}{l}\text { Participants with good sleep quality } \\
\qquad(\mathrm{n}=94)\end{array}$ & $\begin{array}{l}\text { Participants with poor sleep quality } \\
\qquad(\mathrm{n}=42)\end{array}$ & p-value \\
\hline Age (years) & $35.0 \pm 10.7$ & $36.8 \pm 11.7$ & 0.388 \\
\hline Male sex & $29(30.9)$ & $13(31.0)$ & 0.991 \\
\hline $\operatorname{BMI}\left(\mathrm{kg} / \mathrm{m}^{2}\right)$ & $23.2 \pm 3.6$ & $24.3 \pm 3.9$ & 0.123 \\
\hline Waist circumference (cm) & $79.7 \pm 11.1$ & $81.5 \pm 11.4$ & 0.402 \\
\hline Systolic blood pressure (mm Hg) & $117.8 \pm 13.0$ & $119.1 \pm 13.9$ & 0.613 \\
\hline Diastolic blood pressure (mm Hg) & $74.3 \pm 10.2$ & $75.0 \pm 9.9$ & 0.723 \\
\hline WBC count $($ cells $/ \mu \mathrm{L})$ & $5,649.4 \pm 1,058.4$ & $6,188.8 \pm 1,458.4$ & 0.016 \\
\hline Insulin $(\mu \mathrm{IU} / \mathrm{mL})$ & $6.4(5.2-9.1)$ & $8.4(5.7-11.8)$ & 0.024 \\
\hline Fasting plasma glucose (mg/dL) & $96.4 \pm 13.7$ & $99.0 \pm 11.9$ & 0.286 \\
\hline HOMA-IR & $1.5(1.2-2.2)$ & $2.1(1.4-2.8)$ & 0.015 \\
\hline Alcohol drinker & $39(41.5)$ & $18(42.9)$ & 0.794 \\
\hline Coffee drinker & $74(78.7)$ & $33(78.6)$ & 0.984 \\
\hline Regular exerciser & $33(35.9)$ & $12(29.3)$ & 0.457 \\
\hline Married & $38(40.4)$ & $21(50.0)$ & 0.329 \\
\hline Having depressive symptoms by BDI score & $9(9.6)$ & $16(38.1)$ & $<0.001$ \\
\hline Medication for hypertension & $4(4.2)$ & $4(9.5)$ & 0.304 \\
\hline Medication for diabetes mellitus & $4(4.2)$ & $3(7.1)$ & 0.661 \\
\hline
\end{tabular}

Data are presented as the mean \pm standard deviation, median (interquartile range), or $\mathrm{n}(\%)$. p-values were obtained using t-test, Mann-Whitney Utest, or chi-squared test. BMI: body mass index, WBC: white blood cell, HOMA-IR: homeostatic model assessment of insulin resistance, BDI: Beck Depression Inventory

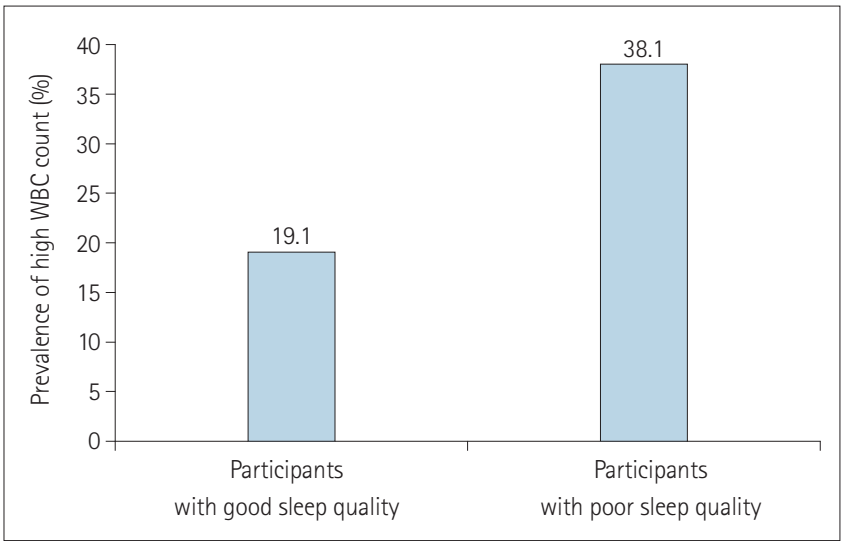

Figure 1. Prevalence of high white blood cell (WBC) count according to sleep quality $(p=0.018)$.

ciations between sleep quality and WBC count remained significant in multiple regression analyses that were adjusted for confounding factors. Compared with the participants with good sleep quality, the participants with poor sleep quality were associated with adjusted $\beta$-coefficient (95\% CIs) for WBC count of $684.1(21.1-1,347.1)$.

Table 3 shows the ORs (95\% CIs) of high WBC count according to sleep quality, determined using multiple logistic regression analyses. In Model 1, the ORs (95\% CIs) were calculated after adjusting for age and sex. In Model 2, we adjusted for additional potential confounding variables, such as alcohol consumption, coffee consumption, physical activity, marital status, hypertension, and diabetes mellitus. In Model 3, we investigated the association between sleep quality and high WBC count, independent of depressive symptoms, obesity effects, and insulin resistance, after
Table 2. Multiple linear regression analysis for the association between sleep quality and WBC count

\begin{tabular}{ccc} 
& $\begin{array}{c}\text { Participants } \\
\text { with good sleep quality } \\
\beta(95 \% \mathrm{CI})\end{array}$ & $\begin{array}{c}\text { Participants } \\
\text { with poor sleep quality } \\
\beta(95 \% \mathrm{CI})\end{array}$ \\
Model 1 & 0 (reference) & $571.8(134.9-1,008.6)$ \\
Model 2 & 0 (reference) & $875.4(297.1-1,452.9)$ \\
Model 3 & 0 (reference) & $684.1(21.1-1,347.1)$ \\
\hline
\end{tabular}

Model 1: adjusted for age and sex. Model 2: adjusted for age, sex, alcohol consumption, coffee consumption, physical activity, marital status, hypertension, and diabetes mellitus. Model 3: adjusted for age, sex, alcohol consumption, coffee consumption, physical activity, marital status, hypertension, diabetes mellitus, depressive symptoms, BMI, and HOMA-IR. WBC: white blood cell, BMI: body mass index, HOMA-IR: homeostatic model assessment of insulin resistance, CI: confidence interval

Table 3. Multiple logistic regression analysis for the association between sleep quality and high WBC count

\begin{tabular}{ccc} 
& $\begin{array}{c}\text { Participants } \\
\text { with good sleep quality } \\
\text { OR (95\% CI) }\end{array}$ & $\begin{array}{c}\text { Participants } \\
\text { with poor sleep quality } \\
\text { OR }(95 \% \text { CI })\end{array}$ \\
Model 1 & 1 (reference) & $2.79(1.22-6.35)$ \\
Model 2 & 1 (reference) & $5.07(1.77-14.52)$ \\
Model 3 & 1 (reference) & $3.83(1.09-13.41)$ \\
\hline
\end{tabular}

Model 1: adjusted for age and sex. Model 2: adjusted for age, sex, alcohol consumption, coffee consumption, physical activity, and marital status, hypertension, and diabetes mellitus. Model 3: adjusted for age, sex, alcohol consumption, coffee consumption, physical activity, marital status, hypertension, diabetes mellitus, depressive symptoms, BMI, and HOMA-IR. WBC: white blood cell, BMI: body mass index, HOMA-IR: homeostatic model assessment of insulin resistance, OR: odds ratio, CI: confidence interval 
adjusting for additional confounding variables, such as having depressive symptoms by BDI score, BMI, and HOMA-IR. Compared with the ORs for individuals with good sleep quality, the ORs (95\% CIs) for high WBC count in individuals with poor sleep quality was 2.79 (1.22-6.35) in Model 1, 5.07 (1.77-14.52) in Model 2, and 3.78 (1.07-13.33) in Model 3.

\section{DISCUSSION}

In the current study, we found that poor sleep quality was significantly associated with a high WBC count in Korean adults after adjusting for potential confounding variables. This association was maintained regardless of the effects of depressive symptoms, obesity, and insulin resistance, even after controlling for having depressive symptoms by BDI score, BMI, and HOMA-IR. While there were some inconsistencies, most of the previous studies have shown a relationship between poor sleep quality and inflammatory markers. A meta-analysis of 72 studies reported that greater sleep disturbance was related to higher levels of circulating inflammatory markers, such as IL-6 and CRP [10]. Our findings are consistent with the results of these previous studies, showing an association between poor sleep quality and inflammatory markers. Moreover, our results suggest that poor sleep quality is associated with a high WBC count, a test which is affordable, easy to interpret, and ordered routinely in clinical practice. A previous study revealed a relationship between sleep quality and CRP in Korean adults [8]. However, CRP, the inflammatory marker used in that study, is not routinely measured in clinical practice. Furthermore, the questionnaire used in that study for assessing sleep quality was composed of only two questions, while the present study used the PSQI-K, which is widely used for its reliability and validity for assessing sleep quality [21]. Thus, our results expand on earlier findings regarding the association between sleep quality and inflammatory markers.

Several possible mechanisms could underlie the significant association between sleep quality and high WBC count, one of the inflammatory markers. The sympathetic nervous system (SNS) and hypothalamic-pituitary-adrenal (HPA) are the major effector systems related to sleep and inflammatory markers [22]. SNS activity decreases markedly during sleep [23], accompanied by vagal outflow or a shift from sympathetic to parasympathetic with sleep onset [24]. The nocturnal fall in SNS activity is hindered when sleep disturbance occurs, resulting in an overall increase in SNS activity during the night [18], and these alterations are carried over into the daytime $[25,26]$. Individuals with insomnia also experience increased sympathetic outflow accompanied with increased levels of circulating adrenaline and noradrenaline, which is also related to increased levels of inflammatory markers [27]. In addition to SNS, HPA has an effect on the systems that link sleep and inflammatory markers. Sleep disturbances lead to activation of the HPA axis $[28,29]$. Consequently, repeated HPA axis activation can generate glucocorticoid-resistant immune cells [30-32]. Thus, both inflammation and HPA axis activation occur in peo- ple with sleep disturbances [33].

Another important factor to consider in the context of inflammation is insulin resistance. Insulin resistance is increasingly being perceived as a chronic low-grade inflammatory status, and poor sleep quality is associated with insulin resistance [34,35]. Indeed, in this study, median value of HOMA-IR (a measure for quantifying insulin resistance) was significantly higher in individuals with poor sleep quality than in those with good sleep quality. Based on this evidence, the interrelationship between sleep quality and inflammation may be explained by insulin resistance.

This study had several limitations. First, this study used a crosssectional design. Therefore, caution should be exercised in causal and temporal interpretations, and additional longitudinal studies are needed. Second, sleep quality was evaluated using a subjective tool instead of an objective tool, such as polysomnography. However, the PSQI-K is a reliable and valid questionnaire for assessing sleep quality and can be useful for differentiating between individuals with good sleep quality and poor sleep quality [21]. Third, only one WBC count measurement was included in the analysis; thus, it was not possible to determine whether an acute and brief episode of infection affected the findings reported herein. To minimize this limitation, subjects with a WBC count $<4,000$ cells $/ \mu \mathrm{L}$ or $>10,000$ cells $/ \mu \mathrm{L}$ were excluded to minimize this limitation. Fourth, although WBC count can be affected by stress, we did not measure the stress levels of participants. Fifth, when we considered alcohol consumption, we considered only the frequency of alcohol intake. Further studies are needed to consider the amount of alcohol consumed along with the frequency of intake. Sixth, since participants were enrolled in the two hospitals, WBC counts were measured with two hematology analyzers. However, these analyzers showed a good concordance for the basic blood count parameters [36]. Seventh, this study used only WBC count as an inflammatory marker. Further studies are warranted to investigated the association between sleep quality and various inflammatory marker, including CRP. Lastly, the small number of participants may not have been representative of the general population in Korea. Despite these limitations, we believe that this is the first study to demonstrate an association between poor sleep quality and high WBC count. Furthermore, a wide range of confounding factors closely related to poor sleep quality and subclinical inflammation, including age, sex, alcohol consumption, coffee consumption, physical activity, marital status, hypertension, diabetes mellitus, depressive symptoms, BMI, and HOMA-IR, were adjusted for in multiple logistic regression analyses.

In conclusion, poor sleep quality was positively associated with an increased risk of high WBC count in Korean adults. The present study contributes to our understanding of the association between sleep quality and inflammatory markers. The findings of this study may have clinical implications regarding the public health strategies that prevent chronic low-grade inflammation.

\section{Acknowledgments}

This work was supported by the Technology Innovation Program 
(20002781, Platform for Prediction and Management of Health Risk Based on Personal Big Data and Lifelogging) funded by the Ministry of Trade, Industry \& Energy (MOTIE, South Korea).

\section{Conflicts of Interest}

The authors have no potential conflicts of interest to disclose.

\section{Author Contributions}

Conceptualization: all authors. Data curation: all authors. Formal analysis: all authors. Funding acquisition: Ji-Won Lee. Investigation: all authors. Methodology: all authors. Project administration: Ji-Won Lee. Resources: all authors. Software: all authors. Supervision: Ji-Won Lee. Validation: all authors. Visualization: all authors. Writing_original draft: all authors. Writing_-review \& editing: all authors.

\section{ORCID iDs}

Jae-Min Park (1)

https://orcid.org/0000-0001-8873-8832

Ji-Won Lee (1)

https://orcid.org/0000-0002-2666-4249

\section{REFERENCES}

1. Scrivo R, Vasile M, Bartosiewicz I, Valesini G. Inflammation as "common soil" of the multifactorial diseases. Autoimmun Rev 2011;10:369-374.

2. Bauer ME, Teixeira AL. Inflammation in psychiatric disorders: what comes first? Ann N Y Acad Sci 2019;1437:57-67.

3. Bonaccio M, Di Castelnuovo A, Pounis G, De Curtis A, Costanzo S, Persichillo $\mathrm{M}$, et al. A score of low-grade inflammation and risk of mortality: prospective findings from the Moli-sani study. Haematologica 2016;101: 1434-1441

4. Zee PC, Turek FW. Sleep and health: everywhere and in both directions. Arch Intern Med 2006;166:1686-1688.

5. Medic G, Wille M, Hemels ME. Short- and long-term health consequences of sleep disruption. Nat Sci Sleep 2017;9:151-161.

6. Cho HJ, Seeman TE, Kiefe CI, Lauderdale DS, Irwin MR. Sleep disturbance and longitudinal risk of inflammation: moderating influences of social integration and social isolation in the Coronary Artery Risk Development in Young Adults (CARDIA) study. Brain Behav Immun 2015;46:319-326.

7. Meier-Ewert HK, Ridker PM, Rifai N, Regan MM, Price NJ, Dinges DF, et al. Effect of sleep loss on C-reactive protein, an inflammatory marker of cardiovascular risk. J Am Coll Cardiol 2004;43:678-683.

8. Lee HW, Yoon HS, Yang JJ, Song M, Lee JK, Lee SA, et al. Association of sleep duration and quality with elevated hs-CRP among healthy Korean adults. PLoS One 2020;15:e0238053.

9. D’Antono B, Bouchard V. Impaired sleep quality is associated with concurrent elevations in inflammatory markers: are post-menopausal women at greater risk? Biol Sex Differ 2019;10:34.

10. Irwin MR, Olmstead R, Carroll JE. Sleep disturbance, sleep duration, and inflammation: a systematic review and meta-analysis of cohort studies and experimental sleep deprivation. Biol Psychiatry 2016;80:40-52.

11. Irwin MR, Witarama T, Caudill M, Olmstead R, Breen EC. Sleep loss activates cellular inflammation and signal transducer and activator of transcription (STAT) family proteins in humans. Brain Behav Immun 2015;47:86-92.

12. Irwin MR, Wang M, Campomayor CO, Collado-Hidalgo A, Cole S. Sleep deprivation and activation of morning levels of cellular and genomic markers of inflammation. Arch Intern Med 2006;166:1756-1762.

13. Carroll JE, Carrillo C, Olmstead R, Witarama T, Breen EC, Yokomizo M, et al. Sleep deprivation and divergent toll-like receptor-4 activation of cellular inflammation in aging. Sleep 2015;38:205-211.

14. Chun MY. Validity and reliability of Korean version of International Physical Activity Questionnaire short form in the elderly. Korean J Fam Med 2012; 33:144-151.
15. Beck AT, Steer RA, Ball R, Ranieri W. Comparison of Beck Depression Inventories-IA and -II in psychiatric outpatients. J Pers Assess 1996;67:588597.

16. Jo SA, Park MH, Jo I, Ryu SH, Han C. Usefulness of Beck Depression Inventory (BDI) in the Korean elderly population. Int J Geriatr Psychiatry 2007; 22:218-223.

17. Kim NH, Park JH, Choi DP, Lee JY, Kim HC. Secondhand smoke exposure and depressive symptoms among Korean adolescents: JS high school study. PLoS One 2016;11:e0168754.

18. Kim NH, Kim HC, Lee JY, Lee JM, Suh I. Association between environmental tobacco smoke and depression among Korean women. BMJ Open 2015; 5:e007131.

19. Matthews DR, Hosker JP, Rudenski AS, Naylor BA, Treacher DF, Turner RC. Homeostasis model assessment: insulin resistance and beta-cell function from fasting plasma glucose and insulin concentrations in man. Diabetologia 1985;28:412-419.

20. Buysse DJ, Reynolds CF 3rd, Monk TH, Berman SR, Kupfer DJ. The Pittsburgh Sleep Quality Index: a new instrument for psychiatric practice and research. Psychiatry Res 1989;28:193-213.

21. Sohn SI, Kim DH, Lee MY, Cho YW. The reliability and validity of the Korean version of the Pittsburgh Sleep Quality Index. Sleep Breath 2012;16:803812.

22. Irwin MR. Sleep and inflammation: partners in sickness and in health. Nat Rev Immunol 2019;19:702-715.

23. Irwin M, Thompson J, Miller C, Gillin JC, Ziegler M. Effects of sleep and sleep deprivation on catecholamine and interleukin-2 levels in humans: clinical implications. J Clin Endocrinol Metab 1999;84:1979-1985.

24. Boudreau P, Yeh WH, Dumont GA, Boivin DB. Circadian variation of heart rate variability across sleep stages. Sleep 2013;36:1919-1928.

25. Irwin MR, Ziegler M. Sleep deprivation potentiates activation of cardiovascular and catecholamine responses in abstinent alcoholics. Hypertension 2005;45:252-257.

26. Tobaldini E, Cogliati C, Fiorelli EM, Nunziata V, Wu MA, Prado M, et al. One night on-call: sleep deprivation affects cardiac autonomic control and inflammation in physicians. Eur J Intern Med 2013;24:664-670.

27. Vgontzas AN, Fernandez-Mendoza J, Liao D, Bixler EO. Insomnia with objective short sleep duration: the most biologically severe phenotype of the disorder. Sleep Med Rev 2013;17:241-254.

28. Abell JG, Shipley MJ, Ferrie JE, Kivimäki M, Kumari M. Recurrent short sleep, chronic insomnia symptoms and salivary cortisol: a 10-year follow-up in the Whitehall II study. Psychoneuroendocrinology 2016;68:91-99.

29. Castro-Diehl C, Diez Roux AV, Redline S, Seeman T, Shrager SE, Shea S. Association of sleep duration and quality with alterations in the hypothalamic-pituitary adrenocortical axis: the Multi-Ethnic Study of Atherosclerosis (MESA). J Clin Endocrinol Metab 2015;100:3149-3158.

30. Miller AH, Raison CL. The role of inflammation in depression: from evolutionary imperative to modern treatment target. Nat Rev Immunol 2016;16: 22-34.

31. Li LB, Goleva E, Hall CF, Ou LS, Leung DY. Superantigen-induced corticosteroid resistance of human $T$ cells occurs through activation of the mitogen-activated protein kinase kinase/extracellular signal-regulated kinase (MEK-ERK) pathway. J Allergy Clin Immunol 2004;114:1059-1069.

32. Webster JC, Oakley RH, Jewell CM, Cidlowski JA. Proinflammatory cytokines regulate human glucocorticoid receptor gene expression and lead to the accumulation of the dominant negative beta isoform: a mechanism for the generation of glucocorticoid resistance. Proc Natl Acad Sci U S A 2001; 98:6865-6870

33. Floam S, Simpson N, Nemeth E, Scott-Sutherland J, Gautam S, Haack M. Sleep characteristics as predictor variables of stress systems markers in insomnia disorder. J Sleep Res 2015;24:296-304.

34. Kline CE, Hall MH, Buysse DJ, Earnest CP, Church TS. Poor sleep quality is associated with insulin resistance in postmenopausal women with and without metabolic syndrome. Metab Syndr Relat Disord 2018;16:183-189.

35. Lee J, Choi YS, Jeong YJ, Lee J, Kim JH, Kim SH, et al. Poor-quality sleep is associated with metabolic syndrome in Korean adults. Tohoku J Exp Med 2013;231:281-291.

36. Bruegel M, Nagel D, Funk M, Fuhrmann P, Zander J, Teupser D. Comparison of five automated hematology analyzers in a university hospital setting: Abbott CELL-DYN Sapphire, Beckman Coulter DxH 800, Siemens ADVIA 2120i, Sysmex XE-5000, and Sysmex XN-2000. Clin Chem Lab Med 2015; $53: 1057-1071$ 